\title{
Gradient design of metal hollow sphere (MHS) foams with density gradients
}

\author{
Ying Liu ${ }^{1, *}, \mathrm{He}-\mathrm{Xiang} \mathrm{Wu}^{1}$, Bin Wang ${ }^{2}$ \\ ${ }^{1}$ Department of Mechanics, School of Civil Engineering, Beijing Jiaotong University, Beijing 100044 \\ ${ }^{2}$ School of Engineering and Design, Brunel University, Uxbridge, Middlesex UB8 3PH, UK
}

\section{Corresponding author:}

Ying Liu

Department of Mechanics

School of Civil Engineering

Beijing Jiaotong University

Beijing 100044

Email: yliu5@bjtu.edu.cn

Tel: 86-10-51682094; Fax: 86-10-51682094

This is the post-print version of the final paper published in Composites Part B: Engineering, 43(3), 1346-1352. The published article is available from http://www.sciencedirect.com/science/article/pii/S1359836811005269. Changes resulting from the publishing process, such as peer review, editing, corrections, structural formatting, and other quality control mechanisms may not be reflected in this document. Changes may have been made to this work since it was submitted for publication. Copyright @ 2011 Elsevier B.V. 


\section{ABSTRACTS}

Metal hollow sphere (MHS) structures with a density gradient have attracted increasing attention in the effort to pursue improved energy absorption properties. In this paper, dynamic crushing of MHS structures are discussed. MHS of different gradients are modelled by stacks of hollow spheres of the same external diameter but different wall thicknesses in the crushing direction. Then based on the dynamic performance of MHS structures with uniform density, a crude semi-empirical model is developed, showing the effect of density gradient in energy absorption and protection against dynamic crush. The model provides some guidance in the design of MHS structures. Following this, dynamic responses of density graded MHS foams are analyzed using explicit finite element simulation and the proposed formula. Results show that the simple semi-empirical model can predict the response of density gradient MHS foams, thus be used for the gradient design of MHS structures.

Key words: A: Metal Hollow Sphere Foams; B: Dynamic Behaviour; C: Numerical Analysis 


\section{INTRODUCTION}

Due to the high uniformity of the internal topologies, metallic hollow sphere (MHS) structures are studied as alternative microstructures for improving the properties of low-density metal materials, and are notably preferential in fast moving machine parts for their good flexibility and shock absorption compared to heavier aluminum-alloy counterparts [1, 2]. Further improvement and optimization of their energy absorption capacity is always the target. By using the concept of functionally graded materials, which optimizes performance through controlled property gradients, density graded foams, have been produced to have improved the performance compared with uniform foams [3], which show the advantage that the stress distribution and the energy absorption can be controlled by gradient variations, allowing designers to achieve significantly enhancement in structural safety against impact loading.

Recently, increased effects have been seen to investigate the (quasi-) static [4-7] or dynamic [8-10] properties of density-graded foams. Zeng et al. [11] performed a series of experiments to test the dynamic responses of MHS foams with density gradients. Liu et al. [12] also discussed the dynamic properties of MHS arrays with density gradients. Their results show that through proper design of the density gradient, the performance of foams can be controlled. Under the low velocity impact, the effects of gradient profiles are rather limited; whereas for the high velocity impact, the influence of gradient profiles is profound. However, understanding on the roles of the density gradient and the gradient profile are still limited, and design guidance for such materials is still not available.

The objective of this paper is to study the roles of the density gradient on the dynamic performance of MHS foams under different crushing velocities, aiming to provide a simple-to-use guide for design purpose. The paper is arranged as follows. The model material, foams of metal hollow spheres with the same radius but various density gradient profiles through the control of the wall thicknesses, are described in Section 2. In section 3, the dynamic characteristic of uniform MHS foams is investigated. Based on these results, the relation between the dynamic response of density graded MHS foams and imposed crushing velocities is established in a semi-empirical approach in section 4. Finally, the conclusion is given. 


\section{METHODOLOGY}

For functionally graded MHS foams, gradient variation can be obtained through the use of different bulk materials, or by the adjustment of the spheres' geometrical parameters, or both. In fact, there is a particular interest [11] in the second option, to make such graded metal hollow sphere foams with a gradient in the strength/density profile by selecting the wall thickness of the spheres, while at the same time, keeping the component material and the outer radii of the spheres unchanged, thus other properties, such as the acoustic and thermal properties remain the same.

In the present discussion, interest is focused on the effects of the variation of the sphere wall thickness along the impact direction. As shown in Fig. 1, the graded MHS foams are comprised of three layers of hollow spheres with different wall thicknesses in the crush direction, whilst each layer contains three rows of spheres of the same wall thickness (the layer number and rows of spheres in each layer can be changed according to application demands). All spheres are of the same outer radius. If all spheres have the same wall thickness, the MHS foam has the uniform density. Here we restrict ourselves to the simple cubic lattice as an example of MHS functionally graded material. The influence of the lattice structures on the performance of MHSS could refer to [13].

In order to minimize the size effect [14], the specimen dimensions along the $X$ and $Z$ directions (Fig. 1) is kept at least 6 times of the size of the unit cell (leading to either 6 or 8 spheres in these lengths), and the thickness of each row in the $Y$ (impact) direction 2 times of unit cell size. So in the model used here, the number of spheres in each layer along the $X, Y$, and $Z$ directions is $n_{x} \times n_{y} \times n_{z}=8 \times 3 \times 6$, respectively, bringing the total number of sphere to 432 in a typical model, as shown in Fig. 1.

The general expression of the relative densities of the MHS foams can be expressed as

$$
\Delta \rho=\frac{\rho^{*}}{\rho_{s}}=\frac{4 \pi}{3} \sum_{i=1}^{N} t_{i} \frac{3 R^{2}+t_{i}^{2}-3 R t_{i}}{24 R^{3} n_{x} n_{y} n_{z}}
$$

where $\rho^{*}$ is the effective density of the MHS foams, $\rho_{\mathrm{s}}$ is the density of the bulk material, $N$ the total number of spheres in the model, $t_{i}$ the wall thickness of the $i^{\text {th }}$ sphere. All spheres are of the same outer radius $R=20 \mathrm{~mm}$.

As shown in Fig. 1, the MHS foam is supported by a fixed rigid plate at one end (distal end) in the $X Z$ plane 
and deformed dynamically by a rigid plate in the $Y$ direction with an initial velocity $V$ at the other end (proximal end). The mass of the impact plate is kept large at $2 \times 10^{4} \mathrm{~kg}$ so that the reduction in the velocity of the striking plate is insignificant throughout the process. The spheres are perfectly bonded together and no necking due to bonding is considered in the contact regions. The side surfaces of the model are free of lateral constraints. The top and bottom surfaces are not tied to the two impacting and supportive plates, allowing for possible separation.

An explicit dynamic finite element analysis was conducted using LS-DYNA [15]. Element type Shell163, a 4-node quadrilateral shell element provided by the code was used to discretize the spheres. Five integration points through the sphere wall thickness, as well as a full integral element formation, were adopted for accuracy. Each sphere was modeled with 256 elements, which was shown to be sufficient to produce reliable results through a mesh sensitivity study. The automatic time step calculation option from the code was chosen. All possible contacts were considered during the process of crushing, including self-contact of internal surface. All contacts were assumed friction free.

In order to test the validity of the FE model, dynamic responses of uniform iron MHS foams were first calculated and compared to the experimental results available [16]. Here, the nominal compressive stress $\sigma$ is defined as the ratio of the normal reaction force on the striking plate to the initial cross section areas of the model. The nominal strain is defined as the ratio of the overall compression of the model to its original length along the $Y$ direction. Note that the FE model simulated was an SC lattice while the experiment was on random packing. Fig. 2a shows a simulated stress-strain curve under a crushing velocity $V=8 \mathrm{~m} / \mathrm{s}$, whilst the nominal dynamic plateau stresses at the impact end under different impact velocities are given in Fig. 2b, with the dynamic plateau stress defined as

$$
\sigma_{p}=\int_{\varepsilon_{c r}}^{\varepsilon_{D}} \sigma(\varepsilon) d \varepsilon /\left(\varepsilon_{D}-\varepsilon_{c r}\right) \text {, }
$$

where $\varepsilon_{c r}$ is the strain corresponding to the quasi-static plastic collapse stress $\sigma_{c r}^{q s}$, and $\varepsilon_{D}$ the densification strain. It is seen that the numerical results show a similar trend in terms of impact velocity to that of the experimental ones, but are consistently about $10 \%$ higher than the latter. Apart from the packing differences, the discrepancy may also be partially due to the existence of cohesive materials and defects which weaken the experimental specimens inherently. Nevertheless, the trend in the response is borne out by the model. 


\section{DYNAMIC RESPONSES OF MHS FOAMS WITH UNIFORM DENSITY}

In order to provide a simple formula for the design purpose, the dynamic response of uniform MHS foam is modeled using a pragmatic adaptation of the rigid-plastic-locking theory previously outlined in $[17,18]$. This allows the constants in the shock theory equation to be determined. In our discussion, the bulk material of the MHS foams is aluminum with a density $2700 \mathrm{~kg} / \mathrm{m}^{3}$, and is assumed to be elastic-perfectly plastic. The Young's modulus, yield stress and Poisson's ratio are taken as $E_{s}=69 \mathrm{GPa}, \sigma_{y s}=76 \mathrm{MPa}$, and $v=0.3$, respectively. The influence of the air inside the hollow sphere is not considered.

Figure 3 shows the deformation modes of a uniform MHS foam (wall thickness $0.4 \mathrm{~mm}$ ) at $t=0.4 \mathrm{~s}$ after the impact. It shows clearly a progressive crush through the uniform foam. The nominal stress-strain curves for MHS foams are given in Fig. 4. It illustrates a dominant hardening feature under a low crushing velocity (Fig. 4a), and a constant mean-stress phenomenon (plateau stress) then locking under higher crushing velocity (Fig. $4 \mathrm{~b}$ and $4 \mathrm{c}$ ) with higher velocity for higher plateau stress.

To estimate the plateau stress level, the rigid-perfectly plastic-locking $(R-P-P-L)$ idealization is applied where the quasi-static plastic collapse stress $\sigma_{c r}^{q s}$, the dynamic collapse stress $\sigma_{p}$, and the densification strain $\varepsilon_{D}$ are used. Reid and co-workers $[17,18]$ gave a simple form for the dynamic stress of cellular materials based on one-dimensional shock wave theory,

$$
\sigma_{p}=\sigma_{c r}^{q s}+\frac{\rho^{*} v^{2}}{\varepsilon_{D}}=\sigma_{c r}^{q s}+A v^{2}
$$

where $\rho^{*}$ is the density of the cellular material. $A$ is a parameter equal to $\rho^{*} / \varepsilon_{D}$, with $\varepsilon_{D}$ the densification strain. In order to investigate the plateau stresses of MHS foams, the values for $\sigma_{c r}^{q s}$ and $A$ must be given. The definition of the value of $\varepsilon_{D}$ is debatable. Several authors (e.g. Pattofattto et al., [19]) have commented on the 'parameter identification uncertainty'. Reid et al. $[17,18]$ used the definition by Avalle [20] based on the concept of efficiency, 


$$
\left.\frac{d E(\varepsilon)}{d \varepsilon}\right|_{\varepsilon=\varepsilon_{D}}=0
$$

where $E$, the efficiency parameter of the foam, is defined as the ratio of the absorbed energy up to a given nominal strain divided by the corresponding stress value, as follows [20]:

$$
E=\frac{\int_{0}^{\varepsilon} \sigma(\varepsilon) d \varepsilon}{\sigma} .
$$

The densification strain is the strain at the point corresponding to the maximum efficiency (as indicated by the circle shown in Fig. 6a and b). For (quasi-) static situation, Tan et al. [18] gave an empirical scaling relation of the form

$$
\left.\varepsilon_{D}=0.76 \mid 1-3.17\left(\rho_{0} / \rho_{S}\right)+2.17\left(\rho_{0} / \rho_{S}\right)^{3}\right] .
$$

However, the analysis shows that $\varepsilon_{D}$ is likely to be a velocity-sensitive quantity [21], dependent on the degree of cellular collapse which is most likely affected by the inertia. When the impact velocity is greater than the critical velocity $V_{s}=\sqrt{2 \sigma_{y s} \varepsilon_{D} / \rho^{*}}$, beyond which progressive cell crushing occurs [18], it tends to a certain value. Seen as Fig. 5, the maximum efficiency point (circle in Fig. 5b) does not correspond to the densification. The full densification can however be determined by the last main local maximum point (triangular point in Fig. 5a and b), with the corresponding strain as the densification strain $\varepsilon_{\mathrm{D}}$. A more detailed discussion about the definition of the densification strain, which is warranted by the various opinions seen in the literature, is beyond the scope of this paper, and will be given in a companion paper.

Following the choice of the last main local point in Fig. 5b, the corresponding estimation of the steady dynamic density densification strain $\varepsilon_{\mathrm{D}}$ can be obtained for $V=150 \mathrm{~m} / \mathrm{s}$. Whilst, the static values of the densification strain, marked as $\varepsilon_{\mathrm{DS}}$ in Table 1, are also obtained from Eq. (6) for different ratios of the wall thickness to outer radius. It is seen that $\varepsilon_{\mathrm{DS}}$ is much smaller than the corresponding dynamic densification strain $\varepsilon_{\mathrm{D}}$. Values of parameter $A$ are calculated from Eq. (3), also given in Table 1. By a linear fitting, 
parameter $A$ could be formulated as

$$
A=1.25 \rho^{*}-45
$$

with the numerical values listed as $A_{i}$ in Table 1 .

For the values of $\sigma_{c r}^{q s}$, referring to Sanders and Gibson [22, 23], we have

$$
\sigma_{0 \mathrm{SC}}=0.65\left(\rho^{*} / \rho_{s}\right)^{1.36} \sigma_{y s}
$$

Then the dynamic plateau stresses of uniform MHS foams can be predicted by

$$
\sigma=0.65 \sigma_{y s}\left(\rho^{*} / \rho\right)^{1.36}+\left(1.25 \rho^{*}-45\right) v^{2}
$$

To look at the influence of the foam's relative density, the plateau stress of MHS foams under velocity crushing velocity $V=150 \mathrm{~m} / \mathrm{s}$ is plotted in Fig.6. Results evaluated using Eq. (8) are marked as $\sigma_{\mathrm{Eq}}$, whilst those obtained by FE simulation are denoted by $\sigma_{\mathrm{FEM}}$. As can be seen, results from the FE analysis and Eq. (8) agree well with each other, with the maximum relative difference within 5\%.

The effect of different striking velocities on the plateau stress is plotted in Fig. 7 for the case $t / R=0.02$. It is seen that results of FEA and from Eq. (8) agree well.

\section{DYNAMIC RESPONSES OF MHS FOAMS WITH DENSITY GRADIENTS}

As shown in Fig. 1, a model of density-graded MHS foam (composed by layers of uniform MHS foams) is proposed. Through the adjustment of sphere wall thickness in different layers, the density gradient is controlled. In this section, the performance of density-graded MHS foams is discussed. The sphere wall thicknesses in the three layers are taken as $t_{1}=0.4 \mathrm{~mm}, t_{2}=0.64 \mathrm{~mm}$, and $t_{3}=0.88 \mathrm{~mm}$, respectively. From Eq. (1), the average relative density of the graded MHS foam is 0.0485 . Two gradient profiles $\mathrm{G}_{123}$ and $\mathrm{G}_{321}$ are considered, with the increasing value of the suffix numbers denoting increased wall thickness though the layers. Thus $G_{123}\left(G_{321}\right)$ is a graded structure with an increasing (decreasing) density along the loading direction. 
Figs. 8 and 9 illustrate the 3D views of the deformation modes of density-graded MHS foams at strain $\varepsilon=0.5$, where the nominal strain $\varepsilon$ is defined as the ratio between the overall compressive displacement of the foams and its original length in the $Y$ direction.

The overall deforming mechanisms show that for both gradient profiles, when loaded under a low speed $(5 \mathrm{~m} / \mathrm{s})$, the dynamic deformation is initiated at the interface between the weaker layers $t_{1}$ and $t_{2}$, then concentrated within the weakest layer $t_{1}$. With layer $t_{1}$ having been compacted and becoming stronger, deformation occurs at the next level of strength - the interface of layer $t_{2}$ and $t_{3}$, then in $t_{2}$, and then $t_{3}$. For a higher velocity $(V=50 \mathrm{~m} / \mathrm{s})$, the above progressive mechanism is exhibited in $\mathrm{G}_{123}$, but for $\mathrm{G}_{321}$, deformation starts to occur simultaneously at both the proximal end within the strongest layer $t_{3}$, like a 'shock' phenomenon, and the weakest layer $t_{1}$ at the distal end under a wave reflection effect. Further increase of the crushing velocity $\left(V=150 \mathrm{~m} / \mathrm{s}, V>V_{\mathrm{s}}\right)$ leads to a complete 'shock wave' pattern, with deformation initiated from the proximal end then progressing row by row to the distal end, irrespective of the layout of the gradient.

The calculated reaction stresses from FEA at both the proximal and distal ends (indexed as 'PE' and 'DE' respectively) are plotted in Fig. 10. At the proximal end, three stepped stress plateaus are observed in the nominal stress-strain curves, which are corresponding to the crushing progress from the weakest layer to the strongest one. The step length $\delta_{s}$ is approximated by

$$
\delta_{s i}=\varepsilon_{D} \cdot \Delta l_{i} / L,
$$

where $\Delta l_{i}$ and $L$ are the thickness of each layer and the specimen in the impact direction. It is seen that under a low crushing velocity $(V=5 \mathrm{~m} / \mathrm{s})$, the responses of $\mathrm{G}_{123}$ and $\mathrm{G}_{321}$ are similar (Fig. 10a), as reported by experimental studies [11]. It is also seen the stress levels at both the proximal and distal ends are almost identical, implying a state of equilibrium throughout the impact process, i.e. the weakest layer collapse first, followed by the $2^{\text {nd }}$ weakest layer, and so on. For higher impact velocities $(V=50$ and $150 \mathrm{~m} / \mathrm{s}$ ), the 'shock type' effect becomes progressively dominant. The collapse force of a sphere depends on the sphere wall thickness, the thicker the sphere wall, the larger the collapse force. As shown in Fig. $10 \mathrm{~b}$ and $10 \mathrm{c}$ for $\mathrm{G}_{321}$, a 
high stress level at the beginning corresponds to the deformation of the proximal layer with the largest wall thickness $t_{3}$, which was also observed in experiments [11]. Fig. $10 \mathrm{~b}(V=50 \mathrm{~m} / \mathrm{s})$ is an intermediate situation with a partial shock-type effect. Fig. 10c clearly illustrates the shock effect where the crush progresses clearly from the proximal end to the distal one, irrespective of the layout of the density gradient, resulting in a step-up stress profile for $G_{123}$ and a step-down one for $G_{321}$. It is noticed that for $G_{321}$, the stress at the distal end is at a lower level, which indicates that placing the 'weakest' layer at the output or distal end with the 'strongest' layer at the impact or proximal end will reduce the stress level transmitted to the protected structures at the distal end, a potentially useful feature for protective application. However, it should be noted that at a low crushing velocity, the stress at the distal end is always enhanced in a progressive crushing mode, in both increasing and decreasing density gradient cases.

According to Eq. (8), the dynamic responses of each component uniform layer in density-graded MHS foam are calculated and marked in Fig. 10 by dash-dot lines with the length determined by Eq. (9). It is seen that Eq. (8) predicts well the plateau stress of layered density-graded MHS foams, especially under a higher velocity crush with the shock type effect. It is noticed that for intermediate velocity crush $(V=50 \mathrm{~m} / \mathrm{s})$ of $\mathrm{G}_{321}$, the order of the stress plateau changes due to the transmission from the static state to the shock one (Fig. 10b). But the values for the stress plateaus agree well with the results given by Eq. (8).

Another key indicator for the energy absorption capabilities of materials is the energy density, which is defined as

$$
W_{\mathrm{v}}=\int_{0}^{\varepsilon_{d}} \sigma(\varepsilon) d \varepsilon
$$

Figure 11 displays the energy density for both MHS with gradient $G_{123}$ and $G_{321}$ under different crushing velocities. It is seen that under a low velocity, it shows a negligible effect of gradient profiles. With increased velocity, the influence of the gradient profiles becomes evident. $W_{\mathrm{v}^{-}} \varepsilon$ curves are convex for $\mathrm{G}_{321}$, but concave for $\mathrm{G}_{123}$ (Figs. $11 \mathrm{~b}$ and $11 \mathrm{c}$ ), which results in more energy absorbed in early stages by $\mathrm{G}_{321}$. Seen in Fig. $11 \mathrm{~b}$, at the strain value of 0.4 , about $44 \%$ more energy has been absorbed 
$\left(\delta W=\left(\left.W_{\mathrm{VG}_{321}}\right|_{\varepsilon=0.4}-\left.W_{\mathrm{VG}_{321}}\right|_{\varepsilon=0.4}\right) /\left.W_{\mathrm{vG}_{321}}\right|_{\varepsilon=0.4}\right)$ by $\mathrm{G}_{321}$. Fig. 11c illustrates that when the crushing velocity is increased further, more than $50 \%$ energy is absorbed at the strain 0.4 in $\mathrm{G}_{321}$, which indicates that the increase of the crushing velocity leads to more energy absorption at the early crushing stage. It is also noticed that the total absorbed energy is independent of the gradient profile.

Based on Eqs. (8) and (9), the predicted energy densities are also plotted in Fig. 11 by blue solid and dash dot lines. It is seen that the predicted values agrees well with the numerical results.

The results displayed in Fig 10 indicate that under a higher velocity impact, the responses of layered density-graded MHS foams display stepped plateau characteristics. The order of the stress plateau is determined by the gradient profile from the proximal end to the distal end. The larger the relative density is, the higher the plateau stress is. The plateau profile, the stress value and the plateau length could be well predicted by the responses of the component uniform layers using Eqs. (8) and (9), especially under a higher crush velocity $\left(V>V_{s}\right)$, where of the shock wave theory applies [17, 18]. Since the influence of lattice structures could be ignored [13] under a higher velocity crush, the formula is also available to the MHS foams with other lattice structures.

\section{CONCLUSIONS}

Aiming at providing a strategy for gradient selection in the design of functional MHS structures, the relation between the dynamic responses of density graded MHS foams and uniform component MHS foam layers was investigated. Instead of the detailed evaluation of the shock wave propagation through different density interface, this study focuses on the global response and the overall stress response of graded MHS foams. The results show that the responses of the density-graded MHS foams could be approximately determined by the dynamic behavior of component uniform layers. Based on this observation, a semi-empirical formula is obtained which is proved to be effective to predict the plateau stress. This method provides a simple guideline in gradient design of density graded MHS foams. 


\section{ACKNOWLEDGEMENTS}

The support of the National Science Foundation of China (No. 10972028), and the State Key Laboratory of Explosion Science and Technology (Beijing Institute of Technology) (under project KFJJ11-3M) are acknowledged. The authors are also grateful to Professor Reid for advice and discussion on the shock wave theory, particularly for future work.

\section{REFERENCES}

[1] Andersen O, Waag U, Schneider L, Stephani G, Kieback B. Novel metallic hollow sphere structures. Advanced Eng Mater 2000; 2:192-195.

[2] Augustin C, Hungerbach W. Production of hollow spheres (HS) and hollow sphere structures (HSS). Mater Lett 2009; 63:1109- 1112.

[3] Matsumoto Y, Brothers AH, Dunand DC. Density-graded aluminum foams by the corrosion method. Mater Sci Forum 2007; 539-543 (Part 2): 1903-1908.

[4] Gupta N. A functionally graded syntactic foam material for high energy absorption under compression. Mater Lett 2007; 61: 979-982.

[5] Kishore, Ravi Shankar, S. Sankaran. Gradient syntactic foams: Tensile strength, modulus and fractographic features. Mater Sci Eng A 2005; 412: 153-158.

[6] Nikhil Gupta, William Ricci. Comparison of compressive properties of layered syntactic foams having gradient in microballoon volume fraction and wall thickness. Mater Sci Eng A 2006; 427: 331-342.

[7] Brothers AH, Dunand DC. Mechanical properties of a density-graded replicated aluminum foam. Mater Sci Eng A 2008; 489: 439-443.

[8] Apetre NA, Sankar BV, Ambur DR. Low-velocity impact response of sandwich beams with functionally graded core. Inter J Solids Struct 2006; 43: 2479-2496.

[9] Cui L, Kiernan S, Gilchrist MD. Designing the energy absorption capacity of functionally graded foam materials. Mater Sci Eng A 2009; 507:215-225. 
[10] Amin Ajdari, Hamid Nayeb-Hashemi, Ashkan Vaziri. Dynamic crushing and energy absorption of regular, irregular and functionally graded cellular structures. Inter J Solids Struct 2011; 48: 506-516.

[11] Zeng HB, Pattofatto S, Zhao H, Girard Y, Fascio V. Impact behavior of hollow sphere agglomerates with density gradient. J Mech Sci 2010; 52: 680-688.

[12] Liu Y, Wu HX, Lu GX, Wang B. Dynamic properties of density graded thin-walled metal hollow sphere arrays. Mech Adv Mater Struct, 2011 (accepted).

[13] Liu Y, Wu HX, Zhang XC, Wang B. The influence of lattice structure on the dynamic performance of metal hollow sphere agglomerates. Mech Res Commun, 2011 (under review).

[14] Gibson LG. Mechanical behaviour of metallic foams. Annu Rev Mater Sci 2000; 30: 191-227.

[15] LSTC. LS-DYNA keyword user's manual. Livermore Software Technology Corporation, 2007.

[16] Zhao H, Elnasri I, Abdennadher S. An experimental study on the behaviour under impact loading of metallic cellular materials. Inter J Mech Sci 2005; 47:757-774.

[17] Reid SR, Peng C. Dynamic uniaxial crushing of wood. Inter J Impact Eng 1997; 19: 531-570.

[18] Tan PJ, Reid SR, Harrigan JJ, Zou Z, Li S. Dynamic compressive strength properties of aluminum foams. Part II-shock theory and comparison with experimental data and numerical models. J Mech Phys Solids 2005; 53: 2206-2230.

[19] Pattofatto S, Elnasri I, Zhao H, Tsitsiris H, Hild F, Girard Y. Shock enhancement of cellular structures under impact loading: Part II analysis. J Mech Phys Solids,2007; 55: 2672-2686.

[20] Avalle M, Belingardi G, Montanini, R. Characterization of polymeric structural foams under compressive impact loading by means of energy-absorption diagram. Inter J Impact Eng 2001; 25: 455-472.

[21] Zou Z, Reid SR, Tan PJ, Li S, Harrigan JJ. Dynamic crushing of honeycombs and features of shock fronts, Inter J Impact Eng 2009; 36: 165-176. 
[22] Sanders WS, Gibson LJ. Mechanics of hollow sphere foams. Mater Sci Eng A 2003; 347: 70-85.

[23] Sanders WS, Gibson LJ. Mechanics of BCC and FCC hollow-sphere foams. Mater Sci Eng A 2003; 352: $150-161$. 


\section{A list of Figure and Table Captions:}

Fig. 1 (color on line) Model of MHS foams with density gradients.

Fig. 2 (color on line) (a) Nominal stress versus strain curves for MHS with SC lattice under crushing velocity $V=8 \mathrm{~m} / \mathrm{s}$; (b) Comparison of plateau stresses between the experiment [16] and the FE model.

Fig. 3 (color on line) Deformation modes for uniform MHS foams with SC lattice under different crushing velocity at $t=0.4 s$. (a) $V=5 \mathrm{~m} / \mathrm{s}$; (b) $V=50 \mathrm{~m} / \mathrm{s}$; (c) $V=150 \mathrm{~m} / \mathrm{s}$.

Fig. 4 Nominal stress-strain curves for uniform MHS foams. (a) $V=5 \mathrm{~m} / \mathrm{s}$; (b) $V=50 \mathrm{~m} / \mathrm{s} ;$ (c) $V=150 \mathrm{~m} / \mathrm{s}$.

Fig. 5 (color on line) Response of uniform MHS foam under a crushing velocity $V=5 \mathrm{~m} / \mathrm{s}$ (a) Typical stress-strain curve; (b) efficiency-strain curve.

Fig. 6 Variation of dynamic plateau stresses of uniform MHS foams versus $t / R$ under the crushing velocity $V=150 \mathrm{~m} / \mathrm{s}$.

Fig. 7 Variation of dynamic plateau stresses versus the crushing velocity $(t / R=0.02)$.

Fig. 8 (color on line) Deformation of MHS foams with density profile $\mathrm{G}_{123}$ at $\varepsilon=0.5$. (a) $V=5 \mathrm{~m} / \mathrm{s}$; (b) $V=50 \mathrm{~m} / \mathrm{s}$; (c) $V=150 \mathrm{~m} / \mathrm{s}$. The green layer has the thinnest wall thickness and red the thickest.

Fig. 9 (color on line) Deformation of MHS foams with density profile $G_{321}$ at $\varepsilon=0.5$. (a) $V=5 \mathrm{~m} / \mathrm{s}$; (b) $V=50 \mathrm{~m} / \mathrm{s} ;(\mathrm{c}) V=150 \mathrm{~m} / \mathrm{s}$. The green layer has the thinnest wall thickness and red the thickest.

Fig. 10 Nominal stress-strain curves at then proximal and distal ends for graded MHS foams under different impact velocities: (a) $V=5 \mathrm{~m} / \mathrm{s}$; (b) $V=50 \mathrm{~m} / \mathrm{s}$; (c) $V=150 \mathrm{~m} / \mathrm{s}$.

Fig.11 (color online) Energy density for density-graded MHS foams under different impact velocities. (a) $V=5 \mathrm{~m} / \mathrm{s} ;$ (b) $V=50 \mathrm{~m} / \mathrm{s} ;$ (c) $V=150 \mathrm{~m} / \mathrm{s}$.

Table 1 Values for dynamic responses of uniform MHS arrays. 


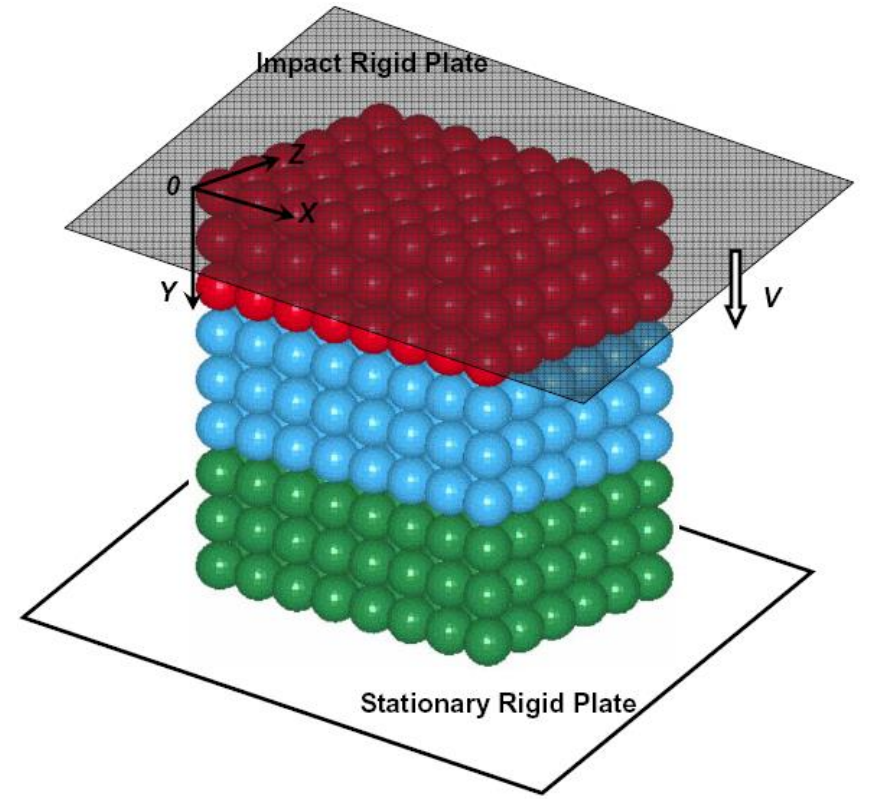

Fig. 1 

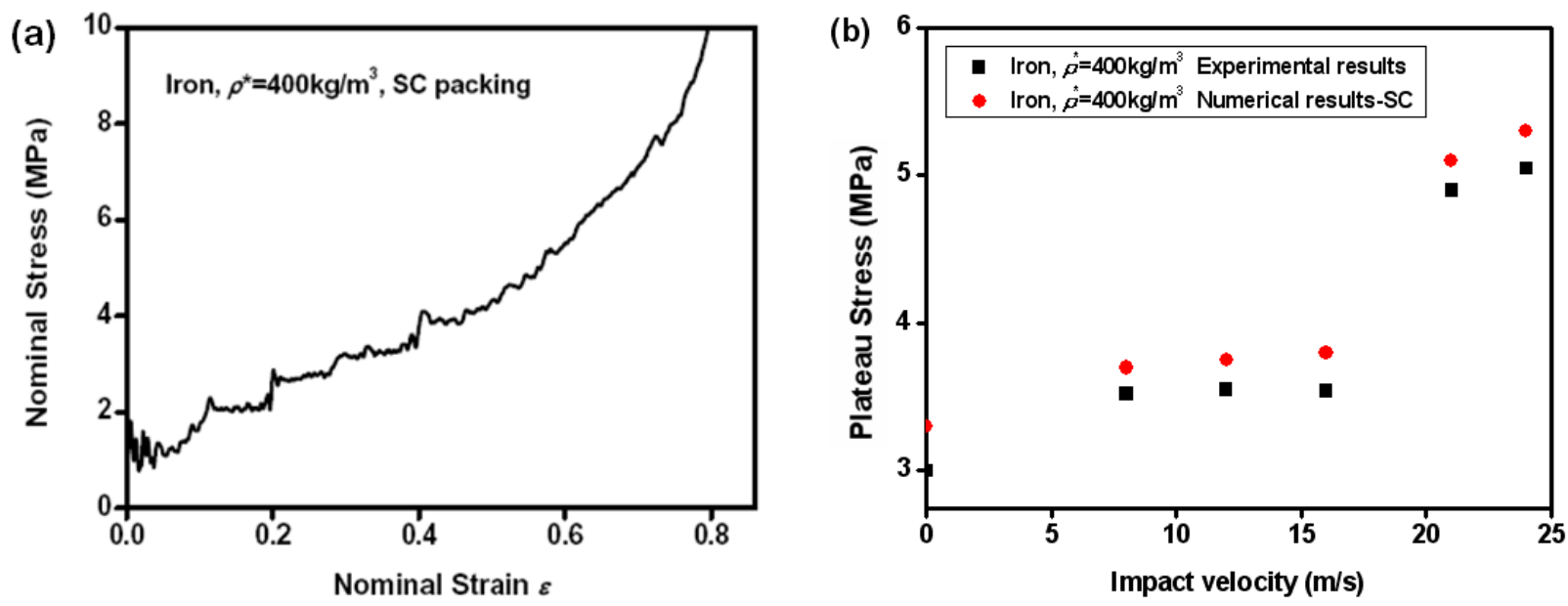

Fig. 2 
(a)

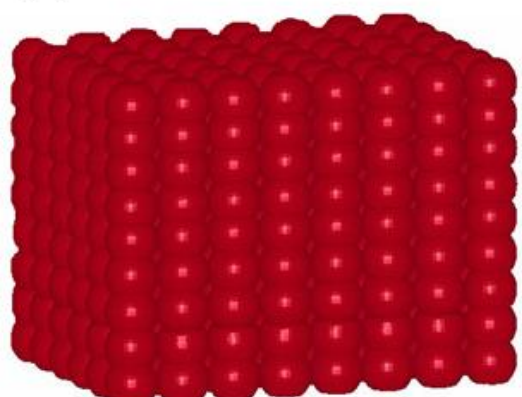

(b)

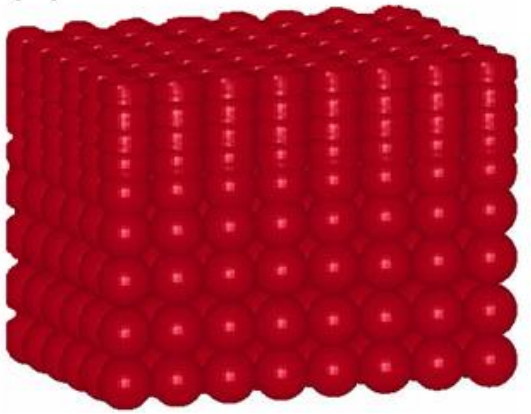

(c)

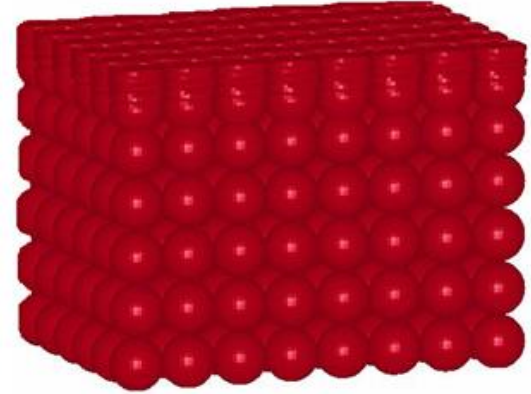

Fig. 3 

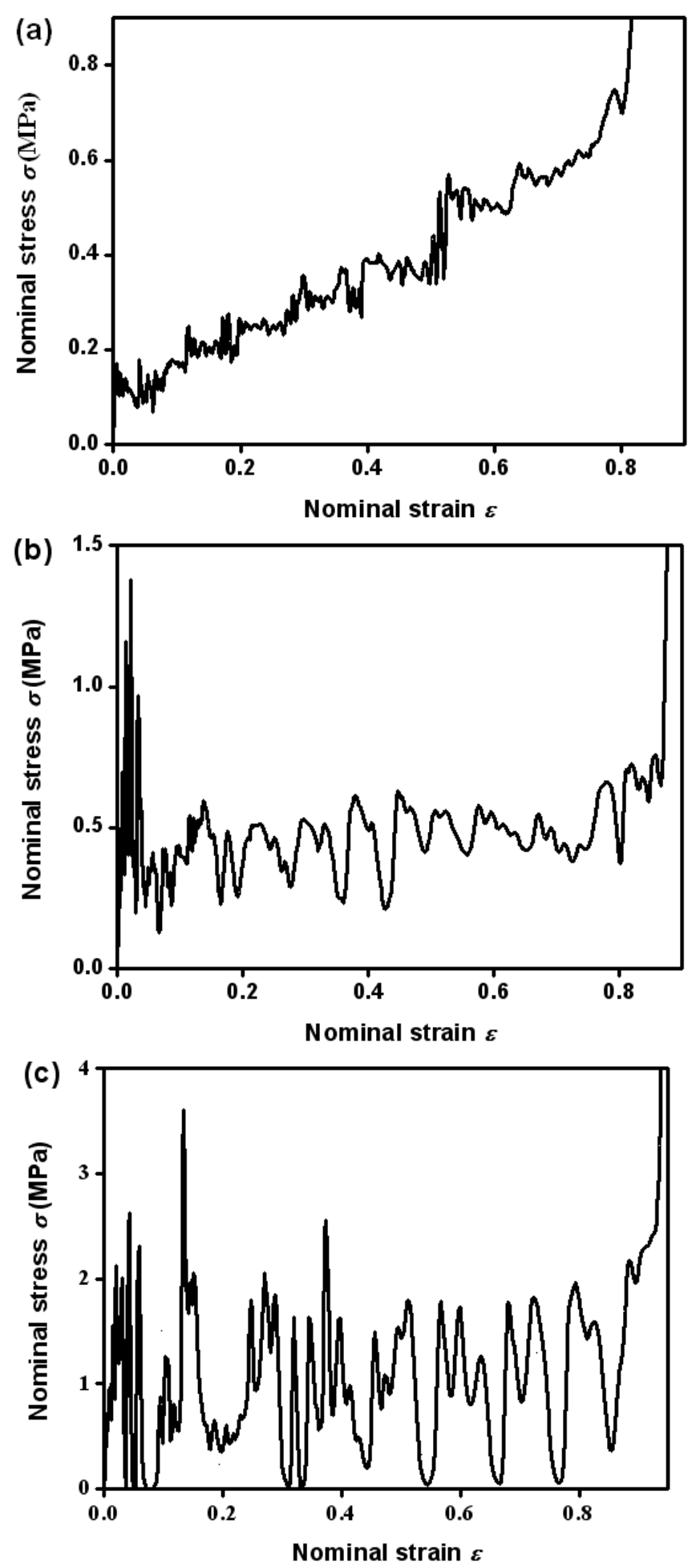

Fig. 4 

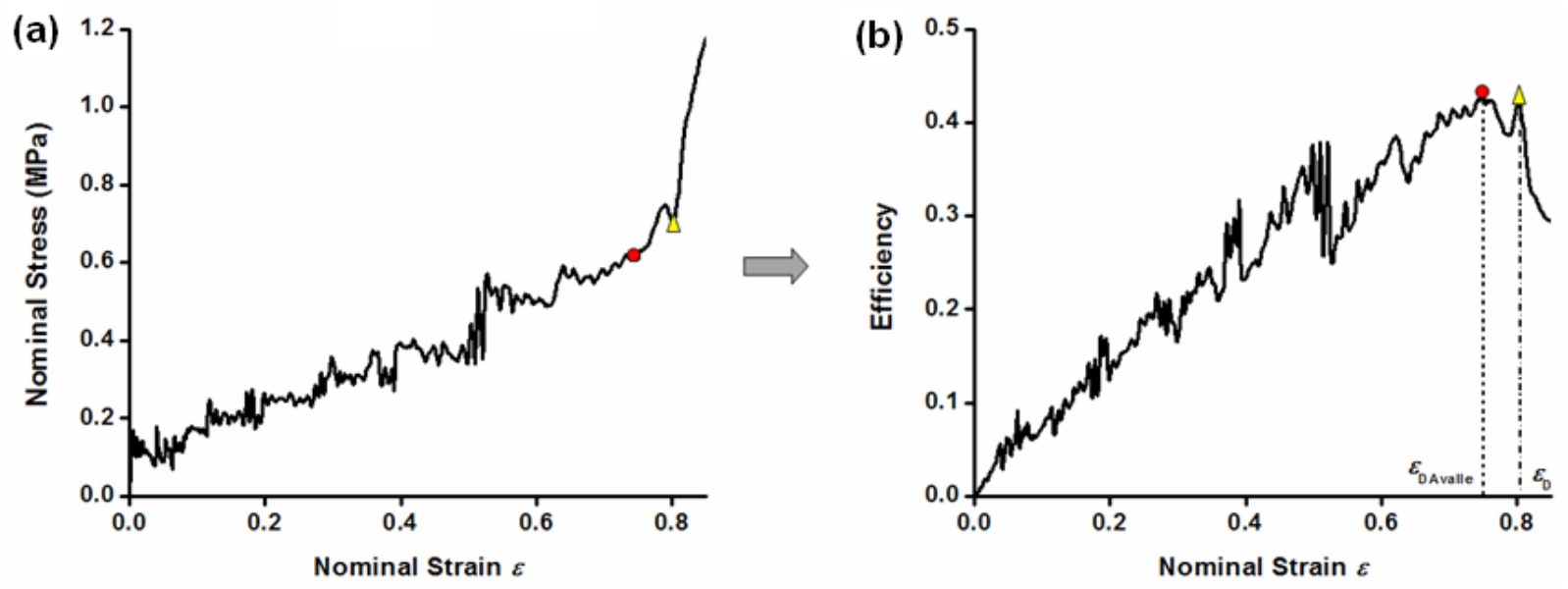

Fig. 5 


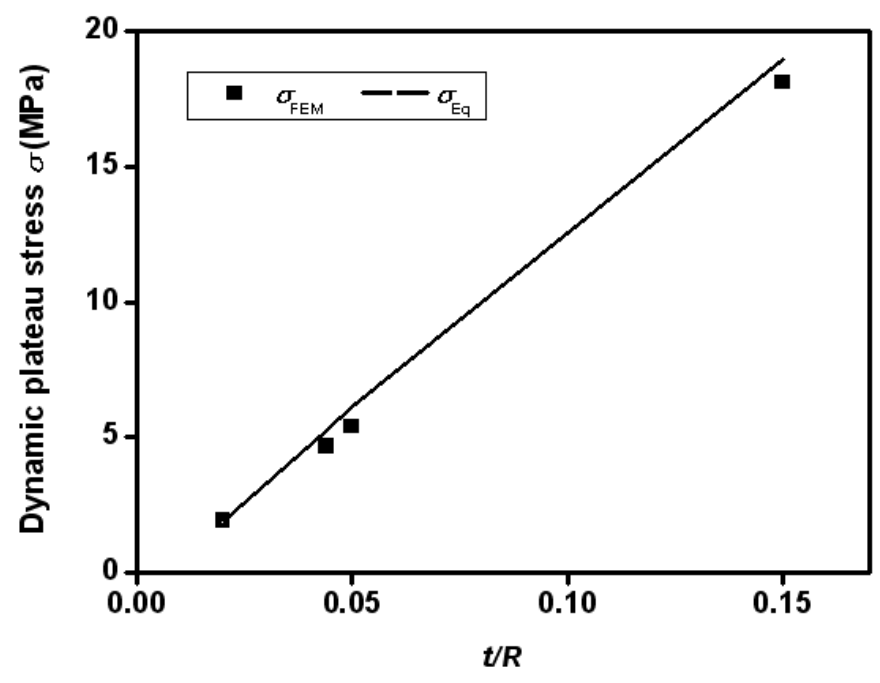

Fig. 6 


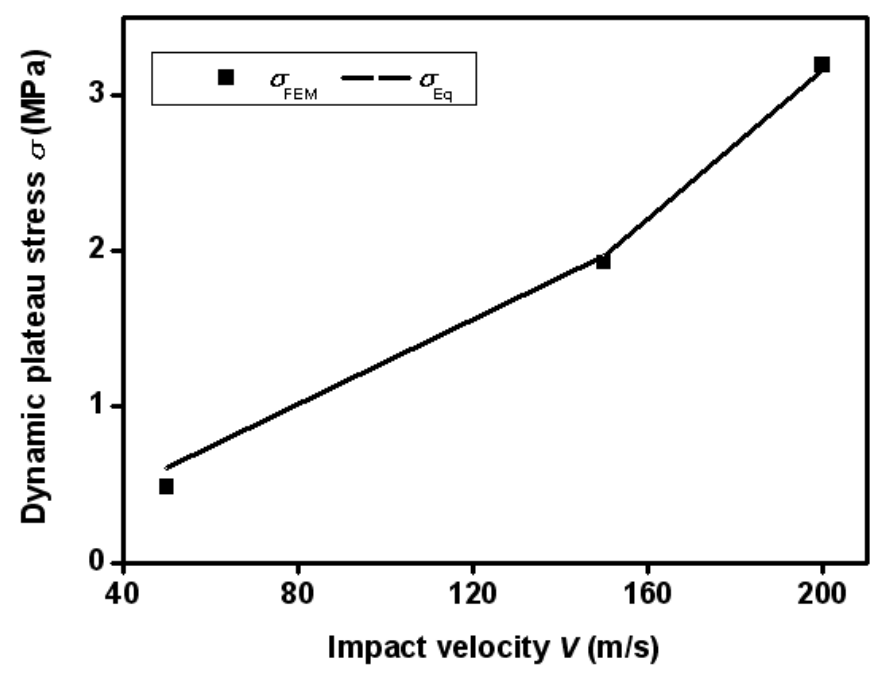

Fig. 7 

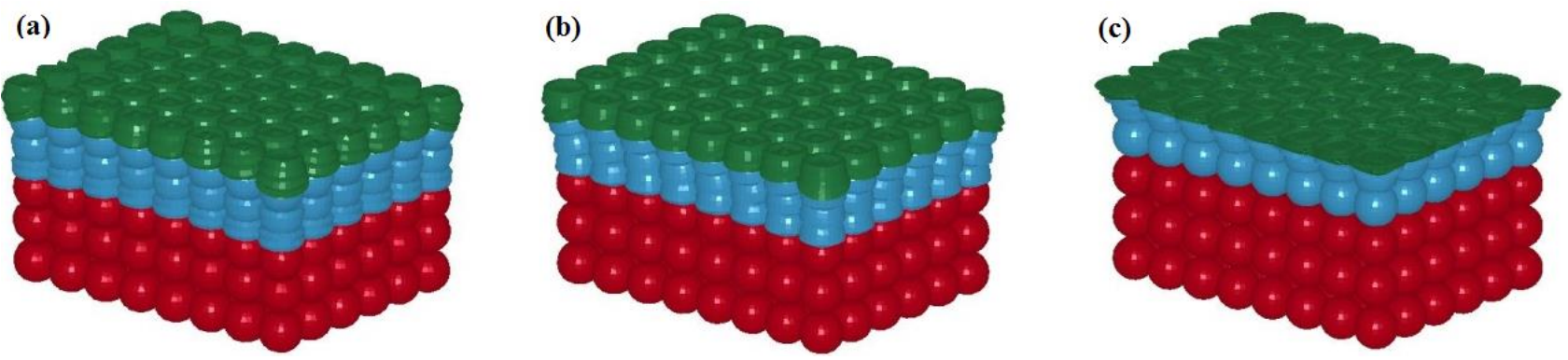

Fig. 8 

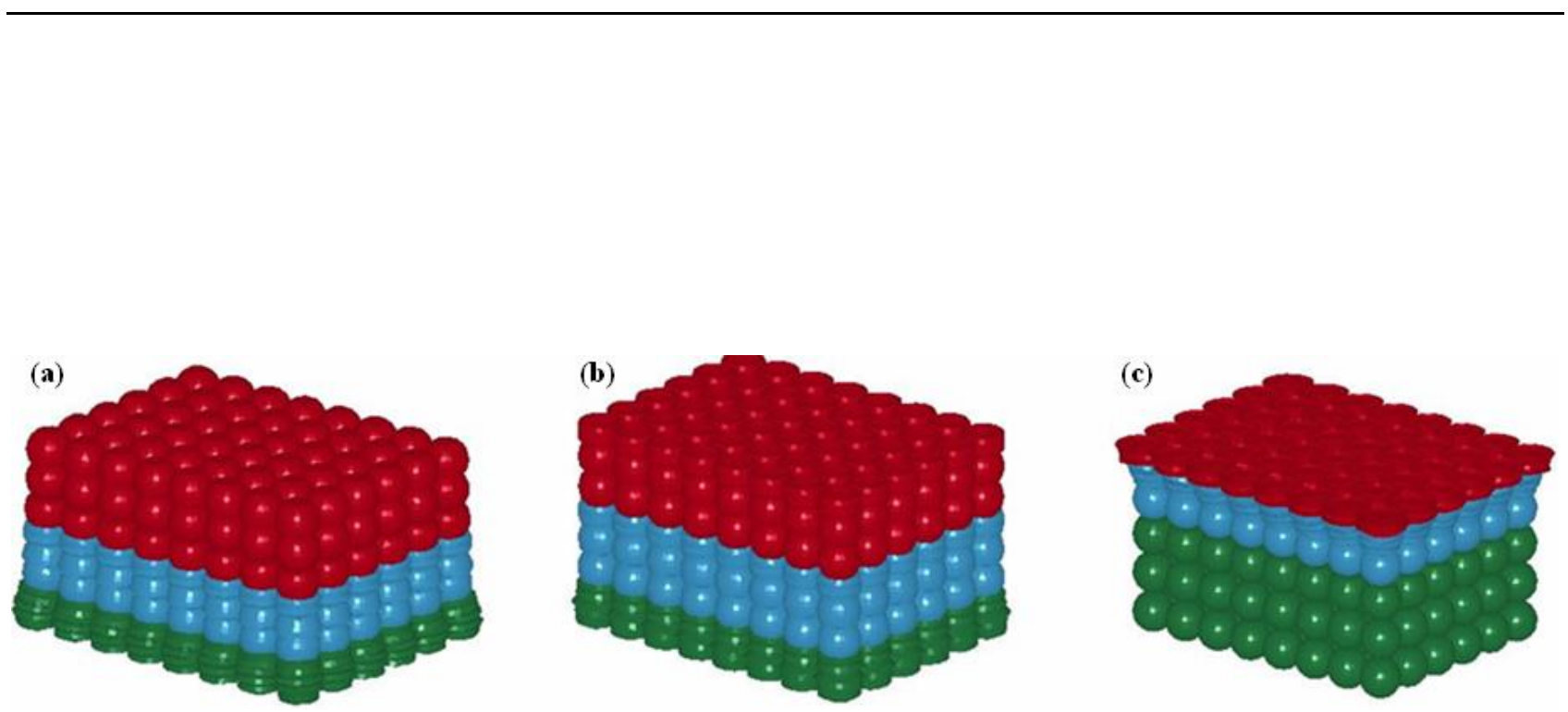

(c)

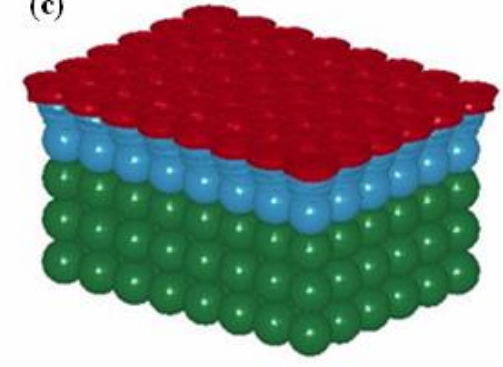

Fig. 9 

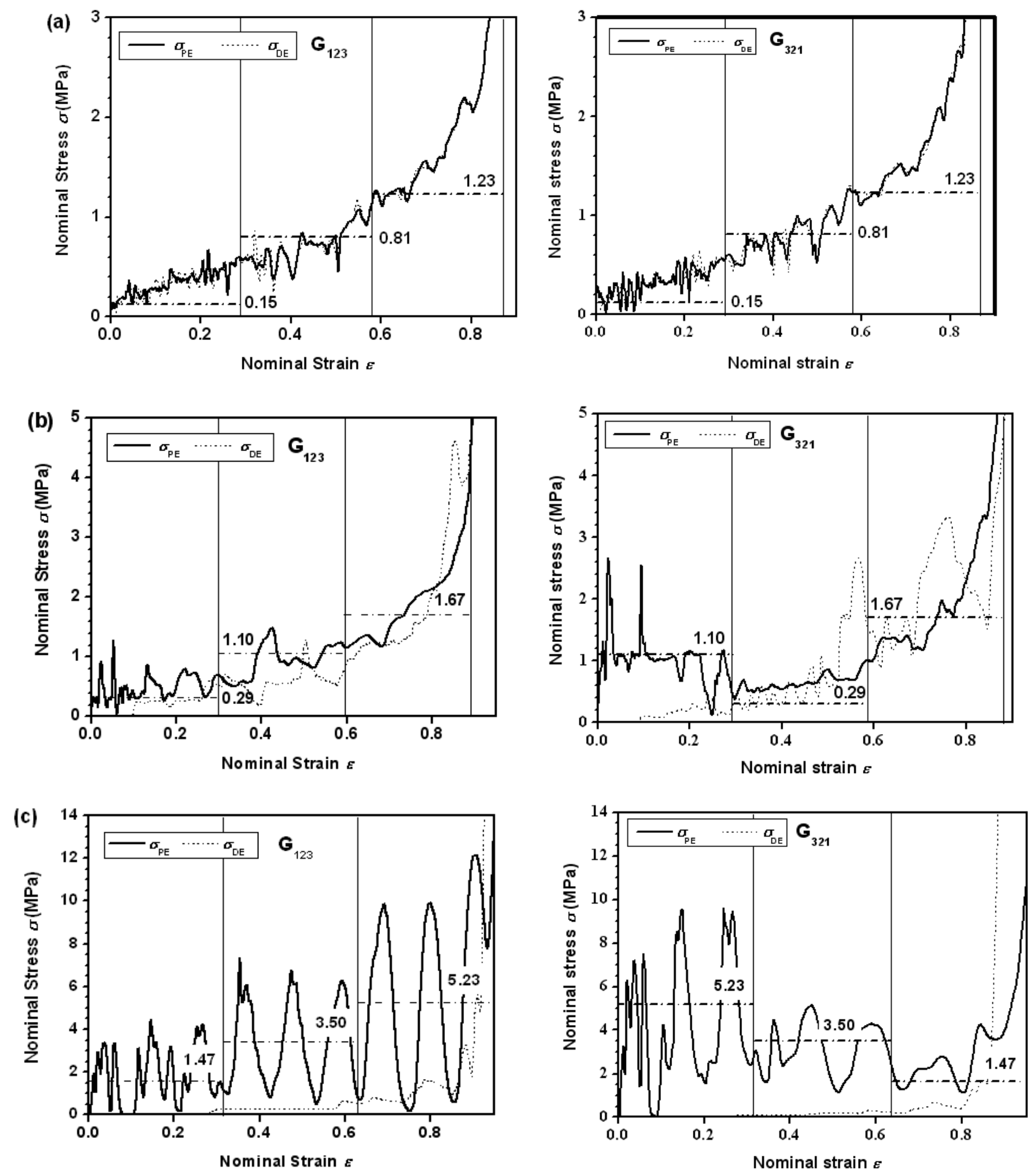

Fig. 10 

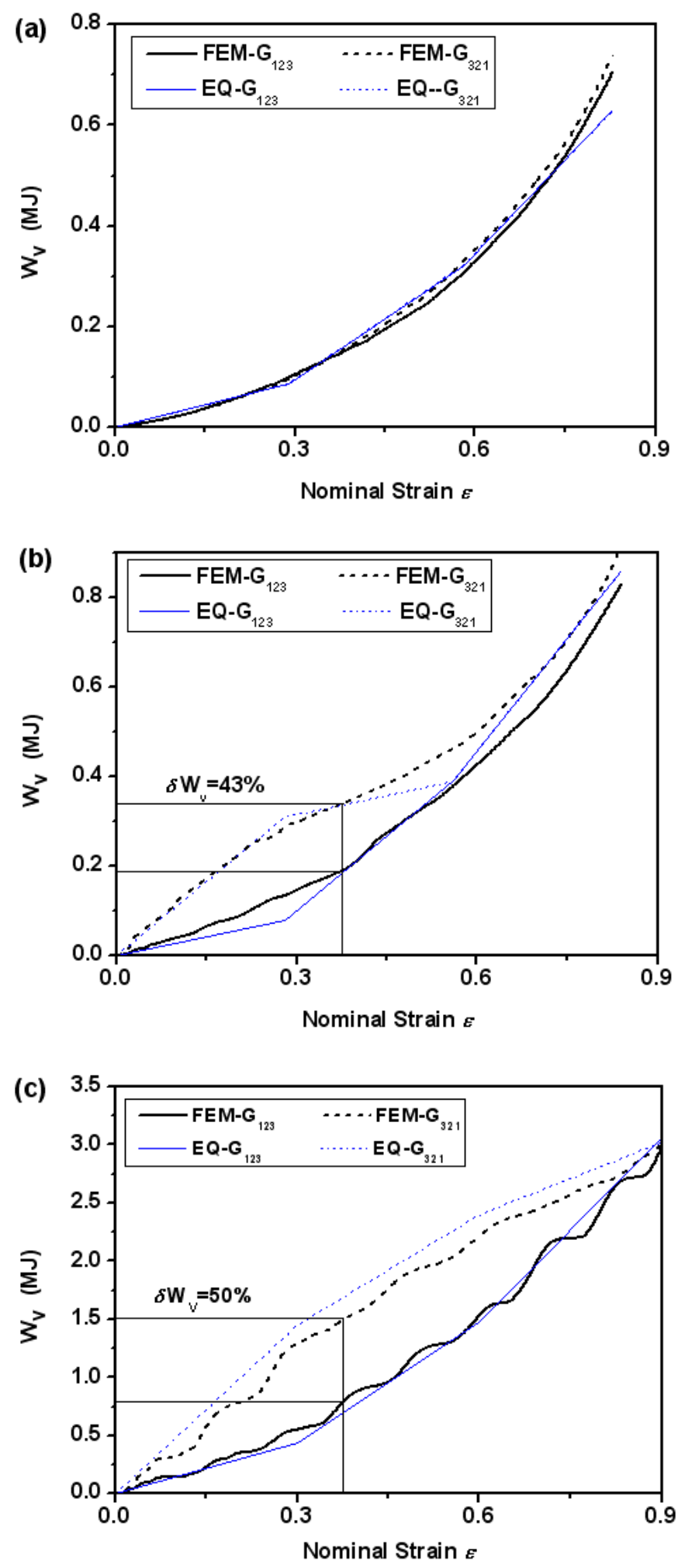

Fig.11 
Table 1

\begin{tabular}{lllllll}
\hline$t / R$ & $\Delta \rho$ & $\rho^{*}$ & $\varepsilon_{\mathrm{D}}$ & $\varepsilon_{\mathrm{DS}}$ & $A$ & $A_{i}$ \\
\hline 0.02 & 0.0308 & 83.13 & 0.95 & 0.686 & 87.5 & 58.9 \\
0.032 & 0.0487 & 131.4 & 0.94 & 0.64 & 139.78 & 119.25 \\
0.044 & 0.0661 & 178.5 & 0.93 & 0.6 & 191.94 & 178.1 \\
0.05 & 0.0747 & 201.6 & 0.92 & 0.58 & 219.13 & 207 \\
0.15 & 0.2020 & 545.5 & 0.79 & 0.28 & 690.5 & 636.9
\end{tabular}

